\title{
Four New Cycloartane Glycosides from Aquilegia vulgaris and Their Immunosuppressive Activities in Mouse Allogeneic Mixed Lymphocyte Reaction
}

\author{
Makiko Nishide, ${ }^{a}$ Hitoshi Yoshimitsu, ${ }^{*}, a$ Masafumi OKawa, ${ }^{b}$ and Toshihiro NoHARA ${ }^{b}$ \\ ${ }^{a}$ Faculty of Engineering, Kyushu Kyoritsu University; 1-8 Jiyugaoka Yahata-nishi-ku, Kitakyushu 807-8585, Japan: and \\ ${ }^{b}$ Faculty of Pharmaceutical Sciences, Kumamoto University; 5-1 Oe-honmachi, Kumamoto 862-0973, Japan. \\ Received February 12, 2003; accepted March 12, 2003
}

Four new cycloartane glycosides, named aquilegiosides $\mathrm{C}-\mathrm{F}$, were isolated from the dried aerial parts of Aquilegia vulgaris. Their structures were determined by two dimensional (2D) NMR spectroscopic analysis and chemical evidence. Aquilegiosides $\mathrm{C}-\mathrm{F}$ suppressed the proliferation of lymphocytes in mouse allogeneic mixed lymphocyte reaction with $\mathrm{IC}_{50}$, ranging from $3.7 \times 10^{-5}$ to $2.2 \times 10^{-4} \mathrm{M}$.

Key words Aquilegia vulgaris; cycloartane glycoside; immunosuppressive activity; aquilegioside; Ranunculaceae

Aquilegia vulgaris L. (Japanese name, seiyouodamaki) is cultivated as a garden plant. We previously reported on the structural elucidation of two cycloartane glycosides, aquilegiosides A and B, from A. flabellata Sieb. et Zucc. var. flabellata (Japanese name, odamaki). ${ }^{1)}$ During our investigation on the chemical constituents in the Ranunculaceous plant, we have now isolated four new cycloartane glycosides, aquilegiosides C (1), D (2), E (3) and F (4), as well as two known ones, aquilegiosides A (5) and B (6), from the dried aerial parts of A. vulgaris. This paper describes their structural elucidation. Immunosuppressive activities of the isolated cycloartane glycosides in mouse allogeneic mixed lymphocyte reaction are also discussed.

\section{Results and Discussion}

The methanolic extract of the air-dried aerial parts of $A$. vulgaris was partitioned into a chloroform-water solvent system. The water-soluble portion was separated by MCI gel CHP20P, octadecyl silica gel (ODS) and silica gel column chromatographies and finally HPLC to give aquilegiosides $\mathrm{C}$ (1), D (2), E (3) and F (4), together with aquilegiosides A (5) and $\mathrm{B}(\mathbf{6})$.

The molecular formula of aquilegioside $\mathrm{C}$ (1) was determined as $\mathrm{C}_{49} \mathrm{H}_{80} \mathrm{O}_{20}$ by high resolution (HR)-FAB-MS showing an $\left[\mathrm{C}_{49} \mathrm{H}_{80} \mathrm{O}_{20} \mathrm{Na}\right]^{+}$ion at $m / z$ 1011.5156. The IR spectra showed a hydroxyl band $\left(3464 \mathrm{~cm}^{-1}\right)$ and a carbonyl band $\left(1718 \mathrm{~cm}^{-1}\right)$. The ${ }^{1} \mathrm{H}-\mathrm{NMR}$ spectra displayed one cyclopropane methylene at $\delta 0.13(\mathrm{~d}, J=4.3 \mathrm{~Hz})$ and $0.52(\mathrm{~d}$, $J=4.3 \mathrm{~Hz}$ ), four quaternary methyls at $\delta 1.10,1.16,1.17$ and 1.34 , two secondary methyls at $\delta 1.13(J=7.3 \mathrm{~Hz})$ and 1.16 $(J=6.7 \mathrm{~Hz})$, three anomeric protons at $\delta 4.94(\mathrm{~d}, J=7.3 \mathrm{~Hz})$, $5.31(\mathrm{~d}, J=7.9 \mathrm{~Hz})$ and $5.39(\mathrm{~d}, J=7.3 \mathrm{~Hz})$. The above data indicated that 1 was a cycloartane triglycoside derivative. Besides, the chemical shifts of the aglycone moiety except for the signals owing to the side-chain moiety and the D-ring in the ${ }^{13} \mathrm{C}$-NMR spectra of $\mathbf{1}$ showed coincidence with those of squarroside I. ${ }^{2)}$ A sequence of connectives through a secondary methyl proton at $\delta 1.13$, a methine proton at $\delta 2.44$ $(\mathrm{m})$, an oxygen-bearing methine proton at $\delta 5.05$ (ddd, $J=5.5,7.9,7.2 \mathrm{~Hz})$ and methylene protons at $\delta 2.99(1 \mathrm{H}, \mathrm{dd}$, $J=5.5,17.7 \mathrm{~Hz})$ and $3.19(1 \mathrm{H}, \mathrm{dd}, J=7.9,17.7 \mathrm{~Hz})$, in turn, was observed in the ${ }^{1} \mathrm{H}-{ }^{1} \mathrm{H}$ correlation spectroscopy (COSY), and their signals could be assigned to the H-21, H20, H-22 and H-23, respectively. Further, the H-20 coupled with a methine proton at $\delta 2.42$ (br s, H-17). The long-range correlations between $\delta 3.19(\mathrm{H}-23)$ and $\delta 211.6(\mathrm{C}-24)$; a secondary methyl proton at $\delta 1.16$ and the carbon signals at $\delta 47.2(\mathrm{CH}), 71.6\left(\mathrm{CH}_{2}\right), 211.6(\mathrm{C}-24)$ indicated that secondary methyl and hydroxy-methyl groups were located at the terminal on the side chain. In addition, the long-range

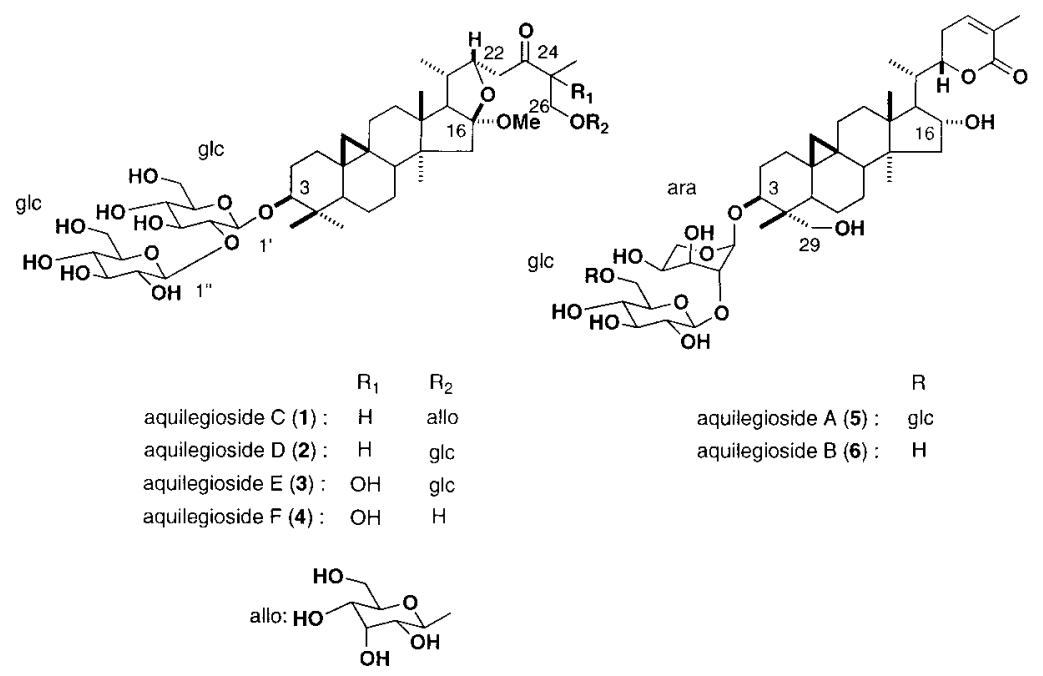


correlation cross peaks between $\delta 2.42(\mathrm{H}-17)$ and $\delta 119.7$ $(\mathrm{C}-16) ; \delta 5.05(\mathrm{H}-22)$ and $\delta 119.7$ (C-16); the methoxy proton at $\delta 3.36$ and $\delta 119.7$ (C-16) resulted in the five-membered acetal ring between C-16 and C-22 (Fig. 1). The nuclear overhauser and exchange spectroscopy (NOESY) and nuclear overhauser effect difference spectra (NOEDS) suggested the stereo configuration for the structure of $\mathbf{1}$ to be as shown in Fig. 2. At the present time, the configuration at C25 is uncertain. On acid hydrolysis, $\mathbf{1}$ afforded D-glucose and D-allose, whose structures were confirmed by the ${ }^{1} \mathrm{H}-\mathrm{NMR}$ coupling pattern (Table 1) and specific rotations using chiral detection in the HPLC analysis. The NMR data could be assigned with the aid of ${ }^{1} \mathrm{H}-{ }^{1} \mathrm{H}$ COSY, heteronuclear multiple quantum coherence (HMQC), total correlation spectroscopy (TOCSY) and heteronuclear multiple bond correlation spectroscopy (HMBC) experiments. In the HMBC experiment, the anomeric proton signals at $\delta 4.94\left(\mathrm{H}-1^{\prime}\right), 5.39\left(\mathrm{H}-1^{\prime \prime}\right)$ and $5.31\left(\mathrm{H}-1^{\prime \prime \prime}\right)$ showed long-range correlations with the carbon signals at $\delta 88.7(\mathrm{C}-3), 83.4\left(\mathrm{C}-2^{\prime}\right)$ and $71.6(\mathrm{C}-26)$, respectively (Fig. 3). From the above evidence, the structure of 1 was concluded to be $26-O-\beta$-D-allopyranosyl $(16 S, 20 S, 22 S)$ $16 \beta, 22$-epoxy- $16 \alpha$-methoxy-3 $\beta, 26$-dihydroxy-cycloartan24 -one 3-O- $\beta$-D-glucopyranosyl-( $1 \rightarrow 2)$ - $\beta$-D-glucopyranoside.

In the ${ }^{1} \mathrm{H}$ - and ${ }^{13} \mathrm{C}$-NMR data (Tables 1,2 ) of aquilegioside $\mathrm{D}(2)\left(\mathrm{C}_{49} \mathrm{H}_{80} \mathrm{O}_{20}\right)$, the signals due to the aglycone moiety and the sugar moiety, attached to the C-3 hydroxyl group, were in good agreement with those of $\mathbf{1}$, although the signals due to the sugar moiety attached to the C-26 hydroxyl group were not identical. On acid hydrolysis, 2 afforded D-glucose and L-arabinose together with several unidentified artificial sapogenols. The above data indicated a glucosyl unit, attached to the C-26 hydroxyl group, in $\mathbf{2}$. The NMR data of $\mathbf{2}$, which could be assigned with the aid of ${ }^{1} \mathrm{H}-{ }^{1} \mathrm{H}$ COSY, HMQC, TOCSY and HMBC techniques, showed signals due to the trisaccharide moiety consisted of three glucopyranosyl moieties [ $\delta 4.88\left(\mathrm{~d}, J=7.9 \mathrm{~Hz}, \mathrm{H}-1^{\prime \prime \prime}\right), 4.95(\mathrm{~d}, J=7.3 \mathrm{~Hz}, \mathrm{H}-$ $\left.1^{\prime}\right)$ and $\left.5.39\left(\mathrm{~d}, J=7.3 \mathrm{~Hz}, \mathrm{H}-1^{\prime \prime}\right)\right]$. The HMBC experiment of 2 showed the same result as that of $\mathbf{1}$ except for the longrange correlation between $\mathrm{H}-1^{\prime \prime \prime}$ of the glucopyranosyl moiety and C-26 of the aglycone moiety. From the data presented above, the structure of $\mathbf{2}$ was concluded to be 26$O$ - $\beta$-D-glucopyranosyl $(16 S, 20 S, 22 S)$-16 $\beta, 22$-epoxy-16 $\alpha$ methoxy-3 $\beta, 26$-dihydroxy-cycloartan-24-one $3-O-\beta$-D-glucopyranosyl- $(1 \rightarrow 2)$ - $\beta$-D-glucopyranoside.

The molecular formula of aquilegioside $\mathrm{E}$ (3) was determined as $\mathrm{C}_{49} \mathrm{H}_{80} \mathrm{O}_{21}$ by HR-FAB-MS showing an $\left[\mathrm{C}_{49} \mathrm{H}_{80} \mathrm{O}_{21} \mathrm{Na}\right]^{+}$ion at $\mathrm{m} / \mathrm{z} 1027.5081$. The ${ }^{1} \mathrm{H}-\mathrm{NMR}$ spectra displayed one cyclopropane methylene at $\delta 0.13(\mathrm{~d}, J=3.7$ $\mathrm{Hz})$ and $0.50(\mathrm{~d}, J=3.7 \mathrm{~Hz})$, five quaternary methyls at $\delta$ $1.09,1.17,1.19,1.35$ and 1.56 , a secondary methyl at $\delta 1.18$ $(J=7.3 \mathrm{~Hz})$, three anomeric protons at $\delta 4.93(\mathrm{~d}, J=7.9 \mathrm{~Hz})$, $4.97(\mathrm{~d}, J=7.3 \mathrm{~Hz})$ and $5.41(\mathrm{~d}, J=7.3 \mathrm{~Hz})$. The ${ }^{1} \mathrm{H}-\mathrm{NMR}$ data are indicative of $\mathbf{3}$ being a cycloartane triglycoside closely related to $\mathbf{2}$. On acid hydrolysis, $\mathbf{3}$ afforded D-glucose together with several unidentified artificial sapogenols. In a comparative study of the ${ }^{1} \mathrm{H}-\mathrm{NMR}$ data of $\mathbf{3}$ with that of $\mathbf{2 , 3}$ showed the quaternary methyl at $\delta 1.56$ instead of the secondary methyl at $\delta 1.19(\mathrm{~d}, J=6.7 \mathrm{~Hz})$ in 2 . Furthermore, a sequence of connectives through a secondary methyl proton at $\delta 1.18(J=7.3 \mathrm{~Hz}, \mathrm{H}-21)$, a methine proton at $\delta 2.53(\mathrm{~m}$,

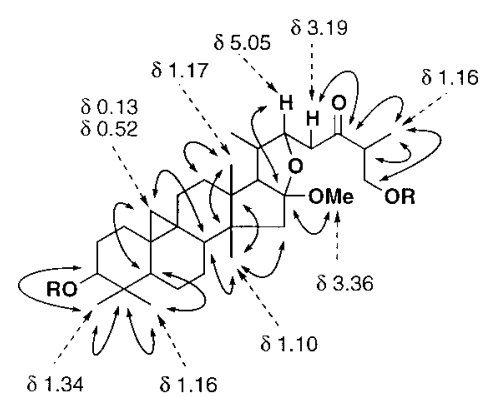

Fig. 1. HMBC Correlations of 1

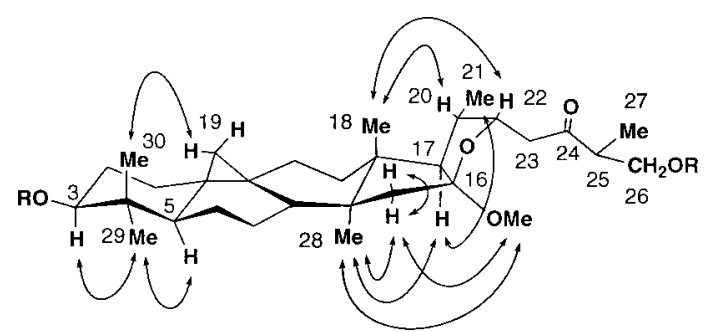

Fig. 2. NOEDS and NOESY Correlations of $\mathbf{1}$
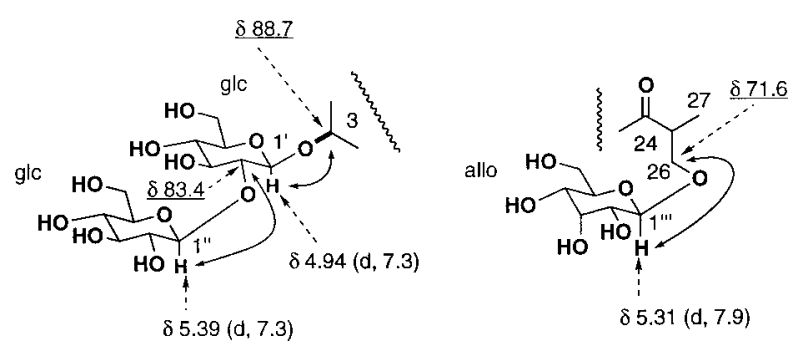

Fig. 3. ${ }^{1} \mathrm{H}-{ }^{13} \mathrm{C}$ Long-Range Correlation of the Saccharide Moieties of $\mathbf{1}$

$J$ values $(\mathrm{Hz})$ in the ${ }^{1} \mathrm{H}$-NMR spectrum are given in parentheses. Underlined values indicate ${ }^{13} \mathrm{C}$-NMR chemical shifts.

$\mathrm{H}-20$ ), a hydroxy-methine proton at $\delta 5.22$ (ddd, $J=6.7,6.7$, $7.3 \mathrm{~Hz}, \mathrm{H}-22)$ and methylene protons at $\delta 3.52(1 \mathrm{H}, \mathrm{dd}$, $J=6.7,18.3 \mathrm{~Hz}, \mathrm{H}-23)$ and $3.60(1 \mathrm{H}, \mathrm{dd}, J=6.7,18.3 \mathrm{~Hz}, \mathrm{H}-$ 23), in turn, was observed in the ${ }^{1} \mathrm{H}-{ }^{1} \mathrm{H}$ COSY. Meanwhile, the molecular formula $\mathrm{C}_{49} \mathrm{H}_{80} \mathrm{O}_{21}$ was higher by $\mathrm{O}_{1}$ than that of 2. The above data indicated the presence of an additional hydroxyl group, linked to the C-25, in $\mathbf{3}$. The long-range correlations between the quaternary methyl proton at $\delta 1.56$ and the carbon signals at $\delta 77.2\left(\mathrm{CH}_{2}, \mathrm{C}-26\right), 79.8(\mathrm{C}, \mathrm{C}-25)$ and 214.3 (C, C-24) elucidated the terminal structure on the side chain. Moreover, the long-range correlations were observed between $\delta 2.44(\mathrm{H}-17)$ and $\delta 119.6(\mathrm{C}-16)$, between $\delta 5.22$ $(\mathrm{H}-22)$ and $\delta 119.6(\mathrm{C}-16)$ and between the methoxy proton at $\delta 3.36$ and $\delta 119.6(\mathrm{C}-16)$. In addition, the NOESY and NOEDS of 3 showed the same result as that of $\mathbf{1}$. At the present time, the configuration at $\mathrm{C}-25$ is uncertain. The NMR data could be assigned with the aid of ${ }^{1} \mathrm{H}-{ }^{1} \mathrm{H}$ COSY, HMQC, TOCSY and HMBC experiments. In the HMBC experiment, the anomeric proton signals at $\delta 4.97\left(\mathrm{H}-1^{\prime}\right), 5.41\left(\mathrm{H}-1^{\prime \prime}\right)$ and $4.93\left(\mathrm{H}-1^{\prime \prime \prime}\right)$ showed long-range correlations with the carbon signals at $\delta 88.7(\mathrm{C}-3), 83.6\left(\mathrm{C}-2^{\prime}\right)$ and $77.2(\mathrm{C}-26)$, respectively. Consequently, the structure of $\mathbf{3}$ was determined to be $26-O$ - $\beta$-D-glucopyranosyl $(16 S, 20 S, 2 S)-16 \beta, 22$-epoxy- $16 \alpha$ methoxy-3 $\beta, 25,26$-trihydroxy-cycloartan-24-one $3-O-\beta$-D- 
Table 1. ${ }^{1} \mathrm{H}-\mathrm{NMR}$ Chemical Shifts of $\mathbf{1}-\mathbf{4}$ (Pyridine- $d_{5}$ )

\begin{tabular}{|c|c|c|c|c|}
\hline & 1 & 2 & 3 & 4 \\
\hline H- 1 & $1.06,1.41$ & $1.07,1.41$ & $1.07,1.41$ & $1.07,1.40$ \\
\hline 2 & $1.83,2.37$ & $1.84,2.38$ & $1.86,2.39$ & $1.86,2.38$ \\
\hline 3 & $3.45 \mathrm{dd}(4.3,11.6)$ & $3.45 \mathrm{dd}(4.9,11.6)$ & $3.46 \mathrm{dd}(4.3,11.5)$ & $3.46 \mathrm{dd}(4.3,11.6)$ \\
\hline 5 & 1.22 & 1.22 & 1.21 & 1.23 \\
\hline 6 & $0.70,1.51$ & $0.69,1.50$ & $0.68,1.50$ & $0.69,1.51$ \\
\hline 7 & $1.01,1.22$ & $0.99,1.21$ & $1.02,1.22$ & $1.01,1.23$ \\
\hline 8 & 1.55 & 1.53 & 1.54 & 1.54 \\
\hline 11 & $1.08,1.91$ & $1.08,1.90$ & $1.08,1.91$ & $1.08,1.90$ \\
\hline 12 & $1.39,1.53$ & $1.38,1.52$ & $1.40,1.53$ & $1.40,1.53$ \\
\hline \multirow[t]{2}{*}{15} & $1.63 \mathrm{~d}(13.4)$ & $1.63 \mathrm{~d}(13.4)$ & $1.65 \mathrm{~d}(12.8)$ & $1.65 \mathrm{~d}(13.4)$ \\
\hline & $1.93 \mathrm{~d}(13.4)$ & $1.93 \mathrm{~d}(13.4)$ & $1.93 \mathrm{~d}(12.8)$ & $1.94 \mathrm{~d}(13.4)$ \\
\hline 17 & $2.42 \mathrm{brs}$ & $2.41 \mathrm{br} \mathrm{s}$ & $2.44 \mathrm{br} \mathrm{s}$ & $2.45 \mathrm{br} \mathrm{s}$ \\
\hline 18 & $1.17 \mathrm{~s}$ & $1.17 \mathrm{~s}$ & $1.19 \mathrm{~s}$ & $1.18 \mathrm{~s}$ \\
\hline \multirow[t]{2}{*}{19} & $0.13 \mathrm{~d}(4.3)$ & $0.13 \mathrm{~d}(4.3)$ & $0.13 \mathrm{~d}(3.7)$ & $0.13 \mathrm{~d}(3.7)$ \\
\hline & $0.52 \mathrm{~d}(4.3)$ & $0.51 \mathrm{~d}(4.3)$ & $0.50 \mathrm{~d}(3.7)$ & $0.52 \mathrm{~d}(3.7)$ \\
\hline 20 & $2.44 \mathrm{~m}$ & $2.41 \mathrm{~m}$ & $2.53 \mathrm{~m}$ & $2.57 \mathrm{~m}$ \\
\hline 21 & $1.13 \mathrm{~d}(7.3)$ & $1.14 \mathrm{~d}(7.3)$ & $1.18 \mathrm{~d}(7.3)$ & $1.19 \mathrm{~d}(7.3)$ \\
\hline 22 & $5.05 \operatorname{ddd}(5.5,7.9,7.2)$ & $5.05 \operatorname{ddd}(5.5,7.2,7.9)$ & $5.22 \operatorname{ddd}(6.7,6.7,7.3)$ & $5.22 \operatorname{ddd}(6.7,6.7,7.3)$ \\
\hline \multirow[t]{2}{*}{23} & $2.99 \mathrm{dd}(5.5,17.7)$ & $3.00 \mathrm{dd}(5.5,17.1)$ & $3.52 \mathrm{dd}(6.7,18.3)$ & $3.51 \mathrm{dd}(6.7,18.3)$ \\
\hline & $3.19 \mathrm{dd}(7.9,17.7)$ & $3.21 \mathrm{dd}(7.9,17.1)$ & $3.60 \mathrm{dd}(6.7,18.3)$ & $3.56 \mathrm{dd}(6.7,18.3)$ \\
\hline 25 & 3.13 & 3.17 & & \\
\hline \multirow[t]{2}{*}{26} & $3.78 \mathrm{dd}(5.5,9.8)$ & $3.87 \mathrm{dd}(5.5,9.8)$ & $4.21 \mathrm{~d}(11.0)$ & $4.04 \mathrm{~d}(11.0)$ \\
\hline & $4.41 \mathrm{dd}(7.3,9.8)$ & $4.43 \mathrm{dd}(7.3,9.8)$ & $4.31 \mathrm{~d}(11.0)$ & $4.49 \mathrm{~d}(11.0)$ \\
\hline 27 & $1.16 \mathrm{~d}(6.7)$ & $1.19 \mathrm{~d}(6.7)$ & $1.56 \mathrm{~s}$ & $1.62 \mathrm{~s}$ \\
\hline 28 & $1.10 \mathrm{~s}$ & $1.10 \mathrm{~s}$ & $1.09 \mathrm{~s}$ & $1.10 \mathrm{~s}$ \\
\hline 29 & $1.34 \mathrm{~s}$ & $1.34 \mathrm{~s}$ & $1.35 \mathrm{~s}$ & $1.35 \mathrm{~s}$ \\
\hline 30 & $1.16 \mathrm{~s}$ & $1.16 \mathrm{~s}$ & $1.17 \mathrm{~s}$ & $1.17 \mathrm{~s}$ \\
\hline \multirow[t]{2}{*}{$\mathrm{OMe}$} & $3.36 \mathrm{~s}$ & $3.36 \mathrm{~s}$ & $3.36 \mathrm{~s}$ & $3.36 \mathrm{~s}$ \\
\hline & glc & glc & glc & glc \\
\hline H- $1^{\prime}$ & $4.94 \mathrm{~d}(7.3)$ & $4.95 \mathrm{~d}(7.3)$ & $4.97 \mathrm{~d}(7.3)$ & $4.97 \mathrm{~d}(7.3)$ \\
\hline $2^{\prime}$ & $4.28 \mathrm{dd}(7.3,9.2)$ & $4.26 \mathrm{dd}(7.3,9.2)$ & $4.27 \mathrm{dd}(7.3,9.2)$ & $4.27 \mathrm{dd}(7.3,9.2)$ \\
\hline $3^{\prime}$ & $4.32 \mathrm{dd}(9.2,9.2)$ & $4.33 \mathrm{dd}(9.2,9.2)$ & $4.37 \mathrm{dd}(9.2,9.2)$ & $4.37 \mathrm{dd}(9.2,9.2)$ \\
\hline $4^{\prime}$ & $4.17 \mathrm{dd}(9.2,9.2)$ & $4.17 \mathrm{dd}(9.2,9.2)$ & $4.19 \mathrm{dd}(9.2,9.2)$ & $4.20 \mathrm{dd}(9.2,9.2)$ \\
\hline $5^{\prime}$ & $3.90 \mathrm{~m}$ & $3.90 \mathrm{~m}$ & $3.90 \mathrm{~m}$ & $3.91 \mathrm{~m}$ \\
\hline \multirow[t]{3}{*}{$6^{\prime}$} & $4.35 \mathrm{dd}(4.9,11.6)$ & 4.34 brd (10.4) & 4.32 brd (10.6) & 4.34 br d (10.4) \\
\hline & $4.53 \mathrm{dd}(2.4,11.6)$ & 4.54 brd (10.3) & 4.63 br d (10.8) & 4.55 br d (10.4) \\
\hline & $\mathrm{glc}$ & $\mathrm{glc}$ & glc & glc \\
\hline H- $1^{\prime \prime}$ & $5.39 \mathrm{~d}(7.3)$ & $5.39 \mathrm{~d}(7.3)$ & $5.41 \mathrm{~d}(7.3)$ & $5.41 \mathrm{~d}(7.3)$ \\
\hline $2^{\prime \prime}$ & $4.13 \mathrm{dd}(7.3,9.2)$ & $4.14 \mathrm{dd}(7.3,9.2)$ & $4.15 \mathrm{dd}(7.3,9.2)$ & $4.16 \mathrm{dd}(7.3,9.2)$ \\
\hline $3^{\prime \prime}$ & $4.28 \mathrm{dd}(9.2,9.2)$ & $4.26 \mathrm{dd}(9.2,9.2)$ & $4.27 \mathrm{dd}(9.2,9.2)$ & $4.27 \mathrm{dd}(9.2,9.2)$ \\
\hline $4^{\prime \prime}$ & $4.33 \mathrm{dd}(9.2,9.2)$ & $4.32 \mathrm{dd}(9.2,9.2)$ & $4.37 \mathrm{dd}(9.2,9.2)$ & $4.37 \mathrm{dd}(9.2,9.2)$ \\
\hline $5^{\prime \prime}$ & $3.92 \mathrm{~m}$ & $3.95 \mathrm{~m}$ & $3.96 \mathrm{~m}$ & $3.95 \mathrm{~m}$ \\
\hline \multirow[t]{3}{*}{$6^{\prime \prime}$} & $4.44 \mathrm{dd}(4.3,11.0)$ & $4.47 \mathrm{dd}(4.3,11.0)$ & 4.48 br d (10.4) & $4.47 \mathrm{brd}(10.5)$ \\
\hline & $4.51 \mathrm{dd}(3.0,11.0)$ & 4.51 brd $(10.5)$ & $4.52 \mathrm{dd}(3.1,11.5)$ & 4.53 brd (10.4) \\
\hline & allo & $\mathrm{glc}$ & $\mathrm{glc}$ & \\
\hline H- $1^{\prime \prime \prime}$ & $5.31 \mathrm{~d}(7.9)$ & $4.88 \mathrm{~d}(7.9)$ & $4.93 \mathrm{~d}(7.9)$ & \\
\hline $2^{\prime \prime \prime}$ & $3.39 \mathrm{dd}(3.1,7.9)$ & $4.00 \mathrm{dd}(7.9,9.2)$ & $4.00 \mathrm{dd}(7.9,9.2)$ & \\
\hline $3^{\prime \prime \prime}$ & $4.70 \mathrm{dd}(2.5,3.1)$ & $4.21 \mathrm{dd}(9.2,9.2)$ & $4.22 \mathrm{dd}(9.2,9.2)$ & \\
\hline $4^{\prime \prime \prime}$ & $4.20 \mathrm{dd}(3.1,9.8)$ & $4.25 \mathrm{dd}(9.2,9.2)$ & $4.26 \mathrm{dd}(9.2,9.2)$ & \\
\hline \multirow{3}{*}{$\begin{array}{l}5 \\
6^{\prime \prime \prime}\end{array}$} & $4.44 \mathrm{~m}$ & $3.95 \mathrm{~m}$ & $3.96 \mathrm{~m}$ & \\
\hline & $4.35 \mathrm{dd}(4.9,11.6)$ & $4.37 \mathrm{dd}(5.5,11.5)$ & $4.39 \mathrm{dd}(5.5,11.6)$ & \\
\hline & $4.50 \mathrm{dd}(2.4,11.6)$ & 4.55 br d (10.4) & 4.57 brd (11.0) & \\
\hline
\end{tabular}

Coupling constants $(J$ in $\mathrm{Hz})$ are given in parentheses.

glucopyranosyl-(1 $\rightarrow 2)-\beta$-D-glucopyranoside.

The molecular formula of aquilegioside $\mathrm{F}$ (4) was determined as $\mathrm{C}_{43} \mathrm{H}_{70} \mathrm{O}_{16}$ by HR-FAB-MS showing an $\left[\mathrm{C}_{43} \mathrm{H}_{70} \mathrm{O}_{16} \mathrm{Na}\right]^{+}$ion at $\mathrm{m} / \mathrm{z}$ 865.4553. In the ${ }^{1} \mathrm{H}$ - and ${ }^{13} \mathrm{C}-$ NMR data (Tables 1 and 2) of 4 , the signals due to the aglycone moiety except for the terminal moiety on the side chain and the sugar moiety, attached to the C-3 hydroxyl group, were in good agreement with those of $\mathbf{3}$. On acid hydrolysis, 4 afforded D-glucose together with several unidentified artificial sapogenols. Meanwhile, the molecular formula $\mathrm{C}_{43} \mathrm{H}_{70} \mathrm{O}_{16}$ was lower by $\mathrm{C}_{6} \mathrm{H}_{10} \mathrm{O}_{5}$ than that of 3 . The above data suggested that 4 lost a glucosyl unit, attached to the $\mathrm{C}$ -
26 hydroxyl group, from 3 . The NMR data of $\mathbf{4}$, which could be assigned with the aid of ${ }^{1} \mathrm{H}-{ }^{1} \mathrm{H}$ COSY, HMQC and HMBC techniques, showed signals due to the disaccharide moiety consisted of two glucopyranosyl moieties $[\delta 4.97(\mathrm{~d}$, $\left.J=7.3 \mathrm{~Hz}, \mathrm{H}-1^{\prime}\right)$ and $\left.5.41\left(\mathrm{~d}, J=7.3 \mathrm{~Hz}, \mathrm{H}-1^{\prime \prime}\right)\right]$. In the HMBC experiment, the anomeric proton signals at $\delta 4.97$ $\left(\mathrm{H}-1^{\prime}\right)$ and $5.41\left(\mathrm{H}-\mathrm{1}^{\prime \prime}\right)$ showed long-range correlations with the carbon signals at $\delta 88.7(\mathrm{C}-3)$ and $83.6\left(\mathrm{C}-2^{\prime}\right)$, respectively. Thus, the structure of $\mathbf{4}$ was determined to be $(16 S, 20 S, 22 S)$-16 $\beta, 22$-epoxy- $16 \alpha$-methoxy- $3 \beta, 25,26$-trihydroxy-cycloartan-24-one 3 - $O$ - $\beta$-D-glucopyranosyl- $(1 \rightarrow 2)-\beta$ D-glucopyranoside. 
Table 2. ${ }^{13} \mathrm{C}$-NMR Data for $\mathbf{1}-\mathbf{4}\left(\right.$ Pyridine- $\left.d_{5}\right)$

\begin{tabular}{|c|c|c|c|c|}
\hline & 1 & 2 & 3 & 4 \\
\hline C- 1 & 31.9 & 31.9 & 32.0 & 32.0 \\
\hline 2 & 29.9 & 29.8 & 29.9 & 29.9 \\
\hline 3 & 88.7 & 88.6 & 88.7 & 88.7 \\
\hline 4 & 41.3 & 41.2 & 41.3 & 41.3 \\
\hline 5 & 47.5 & 47.5 & 47.6 & 47.6 \\
\hline 6 & 20.8 & 20.7 & 20.8 & 20.8 \\
\hline 7 & 26.2 & 26.1 & 26.2 & 26.2 \\
\hline 8 & 47.4 & 47.3 & 47.4 & 47.4 \\
\hline 9 & 19.5 & 19.4 & 19.5 & 19.5 \\
\hline 10 & 26.9 & 26.8 & 26.9 & 26.9 \\
\hline 11 & 26.5 & 26.4 & 26.5 & 26.5 \\
\hline 12 & 31.0 & 30.9 & 31.0 & 31.0 \\
\hline 13 & 44.3 & 44.2 & 44.3 & 44.3 \\
\hline 14 & 49.4 & 49.3 & 49.4 & 49.4 \\
\hline 15 & 45.1 & 45.1 & 45.3 & 45.3 \\
\hline 16 & 119.7 & 119.7 & 119.6 & 119.6 \\
\hline 17 & 69.0 & 69.0 & 69.1 & 69.2 \\
\hline 18 & 19.4 & 19.3 & 19.4 & 19.4 \\
\hline 19 & 30.3 & 30.3 & 30.4 & 30.4 \\
\hline 20 & 34.5 & 34.4 & 34.5 & 34.6 \\
\hline 21 & 18.2 & 18.1 & 18.2 & 18.1 \\
\hline 22 & 82.2 & 82.1 & 82.2 & 82.2 \\
\hline 23 & 45.4 & 45.4 & 41.8 & 42.0 \\
\hline 24 & 211.6 & 211.5 & 214.3 & 215.0 \\
\hline 25 & 47.2 & 47.1 & 79.8 & 81.0 \\
\hline 26 & 71.6 & 71.6 & 77.2 & 69.2 \\
\hline 27 & 13.7 & 13.7 & 23.1 & 22.8 \\
\hline 28 & 19.5 & 19.4 & 19.4 & 19.5 \\
\hline 29 & 25.8 & 25.7 & 25.8 & 25.8 \\
\hline 30 & 15.3 & 15.3 & 15.4 & 15.3 \\
\hline \multirow[t]{2}{*}{$\mathrm{OMe}$} & 50.1 & 50.1 & 50.2 & 50.1 \\
\hline & glc & glc & glc & glc \\
\hline C- $1^{\prime}$ & 104.9 & 104.8 & 105.0 & 105.0 \\
\hline $2^{\prime}$ & 83.4 & 83.3 & 83.6 & 83.6 \\
\hline $3^{\prime}$ & 78.3 & 78.3 & 78.4 & 78.4 \\
\hline $4^{\prime}$ & 71.6 & 71.6 & 71.6 & 71.6 \\
\hline $5^{\prime}$ & 78.0 & 77.9 & 78.0 & 78.0 \\
\hline \multirow[t]{2}{*}{$6^{\prime}$} & 62.8 & 62.7 & 62.8 & 62.8 \\
\hline & glc & glc & glc & glc \\
\hline C- $1^{\prime \prime}$ & 106.0 & 105.9 & 106.2 & 106.2 \\
\hline $2^{\prime \prime}$ & 77.1 & 77.0 & 77.2 & 77.2 \\
\hline $3^{\prime \prime}$ & 78.2 & 78.2 & 78.3 & 78.3 \\
\hline $4^{\prime \prime}$ & 71.7 & 71.7 & 71.7 & 71.7 \\
\hline $5^{\prime \prime}$ & 78.0 & 77.9 & 78.0 & 78.0 \\
\hline \multirow[t]{2}{*}{$6^{\prime \prime}$} & 62.8 & 62.7 & 62.8 & 62.8 \\
\hline & allo & glc & glc & \\
\hline C- $1^{\prime \prime \prime}$ & 102.3 & 104.7 & 105.6 & \\
\hline $2^{\prime \prime \prime}$ & 72.2 & 74.9 & 75.0 & \\
\hline $3^{\prime \prime \prime}$ & 73.1 & 78.5 & 78.8 & \\
\hline $4^{\prime \prime \prime}$ & 69.0 & 71.5 & 71.6 & \\
\hline $5^{\prime \prime \prime}$ & 76.0 & 78.5 & 78.5 & \\
\hline $6^{\prime \prime \prime}$ & 63.0 & 62.7 & 62.7 & \\
\hline
\end{tabular}

Aquilegiosides A (5), B (6), C (1), D (2), E (3) and F (4) were evaluated for their immunosuppressive activity in mouse allogeneic mixed lymphocyte reaction. ${ }^{3)}$ Aquilegiosides $\mathrm{C}-\mathrm{F}(\mathbf{1}-\mathbf{4})$ suppressed the proliferation of lymphocytes and the $50 \%$ inhibitory concentrations $\left(\mathrm{IC}_{50}\right)$ were calculated from the dose-dependent curve. These $\mathrm{IC}_{50}$ were listed in Table 3. Consequently, we found that the cycloartane glycosides (1-4), possessing a five-membered acetal ring, had potent suppressive effect against lymphocytes in mice spleen, while the cycloartane glycosides $(\mathbf{5}, \mathbf{6})$ did not show this effect. These results suggested that a five-membered acetal ring of a cycloartane glycoside is essential for expression of the immunosuppressive effect. Moreover, the presence of
Table 3. Inhibitory Effects on Mouse Allogeneic Mixed Lymphocyte Reaction of $1-6$

\begin{tabular}{lcc}
\hline \hline Compounds & $\mathrm{IC}_{50}(\mu \mathrm{g} / \mathrm{ml})$ & $\mathrm{IC}_{50}(\mu \mathrm{M})$ \\
\hline Cyclosporine A & 0.05 & 0.04 \\
Aquilegioside A (5) & $>1000$ & \\
Aquilegioside B (6) & $>1000$ & 227 \\
Aquilegioside C (1) & 225 & 155 \\
Aquilegioside D (2) & 154 & 72 \\
Aquilegioside E (3) & 73 & 37 \\
Aquilegioside F (4) & 31 &
\end{tabular}

an additional hydroxyl group linked to the $\mathrm{C}-25$ and the loss of a glucosyl unit attached to the hydroxyl group at C-26 tended to increase the immunosuppressive effect.

On the other hand, Hemmi et al. reported that cimicifugoside, possessing a five-membered hemiacetal ring, had a suppressive effect on proliferation of mouse lymphocytes stimulated with mitogens such as concanavalin A and lipopolysaccharide. ${ }^{5)}$

\section{Experimental}

Optical rotations were taken with a JASCO DIP-1000 automatic digital polarimeter. The NMR spectra was measured with a JEOL alpha 500 NMR spectrometer and chemical shifts are given on a $\delta(\mathrm{ppm})$ scale with tetramethylsilane (TMS) as an internal standard. The IR spectra was measured with a JEOL JIR-6500W spectrometer. The HR-FAB-MS was recorded with a JEOL HX-110 spectrometer. HPLC was carried out using a TSK gel-120A $(7.8 \mathrm{~mm}$ i.d. $\times 30 \mathrm{~cm})$ column with a Tosoh CCPM pump, Tosoh RI- 8010 detector and JASCO OR-2090 detector. Absorbance spectra was recorded on a Bio-Rad Microplate Reader Model 550. TLC was performed on pre-coated Kieselgel $60 \mathrm{~F}_{254}$ (Merck), and detection was achieved by spraying with $10 \%$ $\mathrm{H}_{2} \mathrm{SO}_{4}$ followed by heating. Column chromatography was carried out on Kieselgel (230 - 400 mesh, Merck), Sephadex-LH20 (Pharmacia Find Chem. Co. Ltd.) and MCI gel CHP20P (Mitsubishi Chemical Ind.)

Plant Material The plant seeds defined as the seed of A. vulgaris were provided by Sakata Seed Corp., Kanagawa, Japan. The plant seeds were cultivated at the Botanical Garden of Kumamoto University.

Extraction and Isolation The dried aerial parts of $A$. vulgaris $(1.3 \mathrm{~kg})$ were extracted with $\mathrm{MeOH}$ at room temperature for six months, and the extract $(308 \mathrm{~g})$ was partitioned in chloroform and water $(1: 1)$. The water-soluble portion (221 g) was subjected to MCI gel CHP20P column chromatography with $\mathrm{MeOH}-\mathrm{H}_{2} \mathrm{O}(50 \rightarrow 60 \rightarrow 70 \rightarrow 80 \rightarrow 90 \%)$ to afford ten fractions (fr. 1 -fr. 10). Fraction 5 ( $3 \mathrm{~g}$ ) was further separated by ODS column chromatography with $\mathrm{MeOH}-\mathrm{H}_{2} \mathrm{O}(40 \rightarrow 50 \rightarrow 60 \rightarrow 70 \%)$ to afford two fractions (fr. 11-fr. 12). Fraction 11 was subjected to silica gel column chromatography with $\mathrm{CHCl}_{3}-\mathrm{MeOH}-\mathrm{H}_{2} \mathrm{O}(7: 3: 0.5)$, followed by $\mathrm{HPLC}$ with $\mathrm{MeOH}-$ $\mathrm{H}_{2} \mathrm{O}(13: 9)$, to furnish aquilegioside E (3) $(8 \mathrm{mg})$. Fraction $6(843 \mathrm{mg})$ was further separated by ODS column chromatography with $\mathrm{MeOH}-\mathrm{H}_{2} \mathrm{O}(40 \rightarrow$ $50 \rightarrow 60 \rightarrow 70 \%$ ) to afford three fractions (fr. 13-fr. 15). Fraction 14 was subjected to silica gel column chromatography with $\mathrm{CHCl}_{3}-\mathrm{MeOH}-\mathrm{H}_{2} \mathrm{O}$ $(7: 3: 0.5)$, followed by HPLC with $\mathrm{MeOH}-\mathrm{H}_{2} \mathrm{O}(13: 9)$, to furnish aquilegioside D (2) (20 mg). Fraction 15 was subjected to silica gel column chromatography with $\mathrm{CHCl}_{3}-\mathrm{MeOH}-\mathrm{H}_{2} \mathrm{O}(7: 3: 0.5)$, followed by HPLC with $\mathrm{MeOH}-\mathrm{H}_{2} \mathrm{O}(13: 9)$, to furnish aquilegioside $\mathrm{C}(\mathbf{1})(21 \mathrm{mg})$. Fraction 7 (606 $\mathrm{mg}$ ) was further separated by ODS column chromatography with $\mathrm{MeOH}-$ $\mathrm{H}_{2} \mathrm{O}(40 \rightarrow 50 \rightarrow 60 \rightarrow 70 \%)$ to afford two fractions (fr. 16-fr. 17). Fraction 16 was subjected to silica gel column chromatography with $\mathrm{CHCl}_{3}-\mathrm{MeOH}-$ $\mathrm{H}_{2} \mathrm{O}(7: 3: 0.5)$, followed by HPLC with $\mathrm{MeOH}-\mathrm{H}_{2} \mathrm{O}(7: 3)$, to furnish aquilegioside F (4) (7 mg). Fraction 17 was subjected to silica gel column chromatography with $\mathrm{CHCl}_{3}-\mathrm{MeOH}-\mathrm{H}_{2} \mathrm{O}(7: 3: 0.5)$, followed by HPLC with $\mathrm{MeOH}-\mathrm{H}_{2} \mathrm{O}(13: 9)$, to furnish aquilegioside A (5) $(45 \mathrm{mg})$. Fraction 10 was subjected to silica gel column chromatography with $\mathrm{CHCl}_{3}-\mathrm{MeOH}-\mathrm{H}_{2} \mathrm{O}$ $(7: 3: 0.5)$, followed by $\mathrm{HPLC}$ with $\mathrm{MeOH}-\mathrm{H}_{2} \mathrm{O}(7: 3)$, to furnish aquilegioside $\mathrm{B}(\mathbf{6})(27 \mathrm{mg})$

Aquilegioside $\mathrm{C}(\mathbf{1})$ : A white powder, $[\alpha]_{\mathrm{D}}^{25}-28.3^{\circ}(c=1.08$, pyridine $)$. Pos. FAB-MS $(\mathrm{m} / \mathrm{z}): 1011[\mathrm{M}+\mathrm{Na}]^{+}$. HR-FAB-MS $(\mathrm{m} / \mathrm{z}): 1011.5156$ $[\mathrm{M}+\mathrm{Na}]^{+}$(Calcd for $\mathrm{C}_{49} \mathrm{H}_{80} \mathrm{O}_{20} \mathrm{Na}$ 1011.5141). IR (KBr) cm ${ }^{-1}: 3464(\mathrm{OH})$, $1718(\mathrm{C}=\mathrm{O}) .{ }^{1} \mathrm{H}$ - and ${ }^{13} \mathrm{C}$-NMR (pyridine- $\left.d_{5}\right)$ : Tables 1 and 2 .

Aquilegioside D (2): A white powder, $[\alpha]_{\mathrm{D}}^{25}-31.7^{\circ}(c=1.07$, pyridine $)$. 
Pos. FAB-MS $(\mathrm{m} / \mathrm{z}): 1011[\mathrm{M}+\mathrm{Na}]^{+}$. HR-FAB-MS $(\mathrm{m} / \mathrm{z}): 1011.5149$ $[\mathrm{M}+\mathrm{Na}]^{+}$(Calcd for $\mathrm{C}_{49} \mathrm{H}_{80} \mathrm{O}_{20} \mathrm{Na} 1011.5141$ ). ${ }^{1} \mathrm{H}$ - and ${ }^{13} \mathrm{C}-\mathrm{NMR}$ (pyridine$\left.d_{5}\right)$ : Tables 1 and 2.

Aquilegioside E (3): A white powder, $[\alpha]_{\mathrm{D}}^{25}-8.6^{\circ}(c=0.43$, pyridine $)$. Pos. FAB-MS $(\mathrm{m} / \mathrm{z}): 1027[\mathrm{M}+\mathrm{Na}]^{+}$. HR FAB-MS $(\mathrm{m} / \mathrm{z}): 1027.5081$ $[\mathrm{M}+\mathrm{Na}]^{+}\left(\mathrm{Calcd}\right.$ for $\left.\mathrm{C}_{49} \mathrm{H}_{80} \mathrm{O}_{21} \mathrm{Na} 1027.5090\right) .{ }^{1} \mathrm{H}-$ and ${ }^{13} \mathrm{C}-\mathrm{NMR}$ (pyridine$d_{5}$ ): Tables 1 and 2 .

Aquilegioside $\mathrm{F}(4)$ : A white powder, $[\alpha]_{\mathrm{D}}^{25}-2.5^{\circ}(c=0.35$, pyridine $)$. Pos. FAB-MS $(\mathrm{m} / \mathrm{z}): 865 \quad[\mathrm{M}+\mathrm{Na}]^{+}$. HR-FAB-MS $(\mathrm{m} / \mathrm{z}): 865.4553$ $[\mathrm{M}+\mathrm{Na}]^{+}$(Calcd for $\mathrm{C}_{43} \mathrm{H}_{70} \mathrm{O}_{16} \mathrm{Na} 865.4562$ ). ${ }^{1} \mathrm{H}$ - and ${ }^{13} \mathrm{C}-\mathrm{NMR}$ (pyridine$d_{5}$ ): Tables 1 and 2 .

Sugar Analysis A solution of each compound (1, 2, 3 or 4) $(1 \mathrm{mg})$ in $2 \mathrm{~N} \mathrm{HCl}$-dioxane $(1: 1,2 \mathrm{ml})$ was heated at $100^{\circ} \mathrm{C}$ for $1 \mathrm{~h}$. The reaction mixture was diluted with $\mathrm{H}_{2} \mathrm{O}$ and evaporated to remove dioxane. The solution was neutralized with Amberlite MB-3 and passed through a SEP-PAK $\mathrm{C}_{18}$ cartridge to give a sugar fraction. The sugar fraction was concentrated to dryness in vacuo to give a residue, which was dissolved in $\mathrm{CH}_{3} \mathrm{CN}-\mathrm{H}_{2} \mathrm{O}$ $(3: 1,250 \mathrm{ml})$. The sugar fraction was analyzed by HPLC under the following conditions: column, Shodex RS-Pac DC-613 $(6.0 \mathrm{~mm}$ i.d. $\times 150 \mathrm{~mm}$, Showa Denko, Tokyo, Japan); solvent, $\mathrm{CH}_{3} \mathrm{CN}-\mathrm{H}_{2} \mathrm{O}$ (3:1); flow rate, 1.0 $\mathrm{ml} / \mathrm{min}$; column temperature, $70^{\circ} \mathrm{C}$; detection, refractive index (RI) and optical rotation $(\mathrm{OR}) \cdot t_{\mathrm{R}}(\mathrm{min})$ of sugars were as follow. 1: D-allose $7.0(+)$, Dglucose $7.4(+)$. 2: D-glucose $7.4(+)$. 3: D-glucose $7.4(+)$. 4: D-glucose 7.4 $(+)$. [reference: D-allose 7.0 (positive optical rotation: + ), D-glucose 7.4 (positive optical rotation: + )].

Biological Assays Animals: Male BALB/cAnNCrj and C57BL/6NCrj mice were purchased from Japan Charles River Laboratories, Kanagawa, Japan. All animals were used at 5 weeks of age.

Cell Culture Medium: RPMI-1640 (Life Technologies, Inc., Rockville, MD, U.S.A.) was supplemented with $2 \mathrm{~mm}$ L-glutamine, penicillin at $100 \mathrm{U} / \mathrm{ml}$, streptomycin at $100 \mu \mathrm{g} / \mathrm{ml}, 25 \mathrm{~nm}$ HEPES and $\mathrm{NaHCO}_{3}$ at $2 \mathrm{mg} / \mathrm{ml}$. Fetal bovine serum (Life Technologies, Inc., Rockville, MD, U.S.A.) was heat-inactivated at $56^{\circ} \mathrm{C}$ for $30 \mathrm{~min}$ and added to the medium as indicated.

Mouse Allogeneic Mixed Lymphocyte Reaction: Mouse allogeneic mixed lymphocyte reaction was carried out by culturing BALB/c mouse spleen cells $\left(4 \times 10^{5}\right.$ cells, responder) and an equal number of C57BL/6 mouse spleen cells treated with mitomycin C at $40 \mu \mathrm{g} / \mathrm{ml}$ for $30 \mathrm{~min}$ at $37^{\circ} \mathrm{C}$ (stimulator) in $200 \mu \mathrm{l}$ of RPMI-1640 medium containing $5 \times 10^{-5} \mathrm{M} 2$-mercaptoethanol, $10 \%$ fetal bovine serum and a variable amount of test substance. The cells were placed in a 96-well flat-bottomed microtest plate (No. 3072 Falcon, Becton Dickinson, Lincoln Park, NJ) and cultured for $4 \mathrm{~d}$ at $37^{\circ} \mathrm{C}$ in an atmosphere of $5 \% \mathrm{CO}_{2}$. After $4 \mathrm{~d}, 10 \mu \mathrm{l}$ of 2-(2-methoxy-4-nitrophenyl)3-(4-nitrophenyl)-5-(2,4-disulfophenyl)-2 $H$-tetrazolium monosodium salt $(\text { WST-8 })^{4)}$ solution was added to each well and cultured for $1 \mathrm{~d}$ at $37^{\circ} \mathrm{C}$ in an atmosphere of $5 \% \mathrm{CO}_{2}$. The absorbance at a wavelength of $450 \mathrm{~nm}$ was measured with a microplate reader. The results were expressed as $\mathrm{IC}_{50}$ values. WST- 8 assay was performed using a cell counting kit-8 (Dojindo Laboratories).

Acknowledgements We are grateful to Mr. K. Takeda and Mr. T. Iriguti of Kumamoto University for measurement of NMR spectra. We are grateful to Prof. H. Okabe and Dr. T. Nagao in Department of Pharmaceutical Sciences, Fukuoka University, for measurements of HR-FAB-MS.

\section{References}

1) Yoshimitsu H., Nishida M., Hashimoto F., Nohara T., Phytochemistry, 51, 449-452 (1999).

2) Yoshimitsu H., Nishida M., Qian Z.-Z., Lei Z.-H., Nohara T., Chem. Pharm. Bull., 48, 828-831 (2000).

3) Bach F. H., Widner M. B., Bach M. L., Klein J., J. Exp. Med., 136, 1430-1444 (1972).

4) Ishiyama M., Miyazono Y., Sasamoto K., Ohkura Y., Ueno K., Talanta, 44, 1299-1305 (1997).

5) Hemmi H., Ishida N., J. Pharmacobio-Dyn., 3, 643-648 (1980). 